\title{
Aplicaciones de SDN en infraestructura de redes
}

\section{educativas}

\section{Aplicaciones de SDN en infraestructura de redes educativas}

\author{
Miguel Fabricio Bone Andrade. ${ }^{1}$, Jaime Darío Rodríguez Vizuete. ${ }^{2}$, Sandra María Sosa \\ Calero. $^{3}$, \& Luis Alfonso Núñez Freire ${ }^{4}$
}

Recibido: 20-11-2020 / Revisado: 28-11-2020 /Aceptado: 17-12-2020/ Publicado: 02-01-2021

\begin{abstract}
.
DOI: https://doi.org/10.33262/cienciadigital.v5i1.1539

Introduction: Although the SDN originated in the academic world, the academic RENs have not widely implemented SDN in their production network, SDN gives us a new architectural direction that allows the network to be controlled in an intelligent and central way, it also allows to be programmed, using software applications. This helps operators manage the entire network efficiently, comprehensively and consistently, regardless of the underlying network technology. Objective: Determine the parameters to apply SDN correctly in educational network infrastructure Method: In order to answer the proposed research question, a methodological process divided into 2 stages is planned: Theoretical analysis and Analysis of the factors that influence the application of SDN in educational networks. Result: A perspective based on the services of the users of university communication networks is presented, and in turn, based on the research, factors related to SDN are proposed when implementing this type of networks. Conclusions The factors that are added to the design of telecommunications infrastructure when applied to a university network are: Automated management of the network. Management of network updates. Network
\end{abstract}

${ }^{1}$ Universidad Técnica Luis Vargas Torres Sede Santo Domingo de los Tsáchilas, Facultad de Ingeniería, Carrera de Ingeniería en Tecnologías de la Información. Santo Domingo de los Tsáchilas, Ecuador. miguel.bone@utelvt.edu.ec https://orcid.org/0000-0002-8635-1869

2 Universidad Técnica Luis Vargas Torres Sede Santo Domingo de los Tsáchilas, Facultad de Ingeniería, Carrera de Ingeniería en Tecnologías de la Información, Santo Domingo de los Tsáchilas, Ecuador. jaime.rodriguez.vizuete@utelvt.edu.ec, https://orcid.org/0000-0003-1397-718X

${ }^{3}$ Universidad Técnica Luis Vargas Torres Sede Santo Domingo de los Tsáchilas, Facultad de Ingeniería, Carrera de Ingeniería en Tecnologías de la Información, Santo Domingo de los Tsáchilas, Ecuador. sandra.soso.calero@utelvt.edu.ec https://orcid.org/0000-0002-9568-1907

4 Universidad Técnica Luis Vargas Torres Sede Santo Domingo de los Tsáchilas, Facultad de Ingeniería, Carrera de Ingeniería en Tecnologías de la Información, Santo Domingo de los Tsáchilas, Ecuador. luis.nunez@utelvt.edu.ec https://orcid.org/0000-0001-9759-2003 
policy language. Security. Energy efficiency. Network virtualization. Distributed SDN controllers. Network measures, Quality of service and quality of user experience QoE. IoT Devices, Smart University, Network Security.

Keywords: Software Defined Networks, SDN in university networks, telecommunications infrastructure.

\section{Resumen.}

Introducción: Aunque el SDN se originó en el mundo académico, los REN académicos no han implementado ampliamente SDN en su red de producción, SDN nos da un nuevo rumbo arquitectónico que permite a la red ser controlada de manera inteligente y central, también permite ser programada, utilizando aplicaciones de software. Lo cual contribuye a que los operadores administren toda la red de manera eficiente, integral y constante, independientemente de la tecnología de red subyacente. Objetivo: Determinar los parámetros para aplicar SDN correctamente en infraestructura de redes educativas Método: Para poder contestar la pregunta de investigación planteada, se planifica un proceso metodológico dividido en 2 etapas: Análisis teórico y Análisis de los factores que influyen al aplicar SDN en redes educativas. Resultado: Se presentan una perspectiva basada en los servicios de los usuarios de las redes de comunicaciones de universidades, y a su vez en base a la investigación se proponen factores relacionados con SDN al implementar este tipo de redes. Conclusiones Los factores que se añaden al diseño de infraestructura de telecomunicaciones al aplicar a una red universitaria son: Gestión automatizada de la red. Gestión de actualizaciones de la red. Lenguaje de políticas de red. Seguridad. Eficiencia energética. Virtualización de redes. Controladores SDN distribuidos. Medidas de red, Calidad de servicio y calidad de la experiencia del usuario QoE. Dispositivos IoT, en la universidad inteligente, Seguridad de la red.

Palabras claves: Redes Definidas por Software, SDN en redes universitarias, infraestructura de telecomunicaciones.

\section{Introducción.}

Debido a los avances que tienen las telecomunicaciones, existe un crecimiento muy importante en las redes de comunicaciones las cuales facilitan la interacción entre los usuarios, siendo esta una parte primordial para afrontar la saturación que se presentan en los medios de comunicación y luego adaptados a una necesidad específica tomando en cuenta características propias y los servicios que proporcionan. (Santillán Lima, Llanga Vargas, \& Chafla, 2017a) Actualmente la infraestructura de telecomunicaciones de una universidad es de gran importancia debido a que la mayoría de las aplicaciones, y herramientas 
educacionales y administrativas se soportan en esta tecnología. Por lo cual existen grandes esfuerzos para desarrollar mejores tecnologías en base a la investigación de la infraestructura de telecomunicaciones que hay detrás de las Redes educativas y de investigación (REN).

Las Redes educativas y de investigación son proveedores de servicios de red especializados, no comerciales que apoyan los servicios dedicados a las necesidades únicas de las comunidades de investigación, incluidas universidades, institutos de investigación, escuelas, hospitales, bibliotecas, museos y otras instalaciones nacionales (Dyer, 2009). Las REN están permitiendo a los investigadores resolver algunos de los mayores desafíos de la sociedad, por ejemplo, gestión de desastres, agricultura, salud, medio ambiente, climatología, etc., además de minimizar la brecha digital y proporcionar oportunidades para la educación y la formación en línea. Por lo general, los REN son organizaciones colaborativas financiadas con fondos públicos y sin fines de lucro. (Chergarova, 2020).

En concordancia con lo anterior se describen más servicios que pueden proporcionar las REN a la comunidad de investigación, como por ejemplo el ancho de banda bajo demanda (BoD), que permite establecer servicios de conectividad de extremo a extremo (E2E) durante un período de tiempo específico con un ancho de banda garantizado. Por ejemplo, ESNet (2017), una red informática de alta velocidad que presta servicios al Departamento de Energía de los Estados Unidos proporciona BoD a través de los circuitos seguros bajo demanda y el sistema de reserva anticipada (OSCARS, 2020).

Del mismo modo, GÉANT (2020), un REN paneuropeo, ofrece BoD a sus usuarios a través de la herramienta de aprovisionamiento AutoBAHN (2020). El suministro del servicio BoD requiere la utilización de los mecanismos de reserva anticipada (Degermark, Köhler, Pink, \& Schelen, 1995) que se suelen utilizar en redes ópticas y de computación grid. (Charbonneau, N., \& Vokkarane, V. M., 2012). No obstante, la aparición de SDN permite volver a examinar cómo se debe admitir TE y cómo se deben manejar las limitaciones de recursos y tiempo que facilitan las reservas anticipadas.

Aunque el SDN se originó en el mundo académico, los REN académicos no han implementado ampliamente SDN en su red de producción. En 2010, el proyecto internacional OpenFlow in Europe Linking Infrastructure and Applications (OFELIA) reunió a 17 instituciones de investigación de Europa, Estados Unidos y América del Sur para crear un banco de pruebas de red para investigación y experimentación. (Chergarova, V. (2020), Sin embargo, la mayoría de las implementaciones de SDN en REN utilizan grupos de pruebas y no una red de producción. La adopción del paradigma SDN es lenta, a pesar de los beneficios sugeridos de mejorar la eficiencia (Xia, Wen, Foh, Niyato y Xie, 2015). 
SDN nos da un nuevo rumbo arquitectónico que permite a la red ser controlada de manera inteligente y central, también permite ser programada, utilizando aplicaciones de software. Lo cual contribuye a que los operadores administren toda la red de manera eficiente, integral y constante, independientemente de la tecnología de red subyacente.

La formulación de SDN se inició en el mundo académico en 2008 como respuesta a las dificultades que enfrentan los profesionales en la gestión de redes actuales. El problema de los dispositivos de red actuales es que normalmente los fabrican proveedores individuales y combinan las funciones esenciales de reenvío de paquetes junto con software de control patentado para controlar esas funciones. Las interfaces propietarias se utilizan generalmente para configurar los dispositivos para políticas de nivel superior. Como resultado, la gestión de las funciones de red está estrechamente vinculada a los dispositivos de hardware individuales, que a su vez están asociados con proveedores individuales e interfaces propietarias. (Alshnta, Abdollah, \& Al-Haiqi, 2018).

En la última década, los requisitos de la red han cambiado rápidamente en respuesta al tamaño creciente del tráfico de la red y los requisitos de calidad; por lo cual, aumenta la exigencia de los objetivos de un extremo a otro (Hamdan et al, 2020). Las arquitecturas de red convencionales son estáticas y complejas para abordar las condiciones dinámicas de la red. Para permitir que las redes sean adaptativas, se ha explorado un nuevo modelo de red emergente denominado SDN (Li, Meng, \& Kwok, 2016; Nayyer et al., 2019; Latif et al., 2020; Chica et al., 2020; . En esencia, SDN separa el plano de control de la red del plano de reenvío de datos (McKeown et al., 2008).

SDN se muestra como un paradigma emergente que está cambiando la forma en que se administran las redes al separar el plano de control del plano de datos y hacer que las redes sean programables. La separación genera flexibilidad, automatización, orquestación y ofrece ahorros tanto en gastos de capital como operativos. (Thimmaraju et al., 2018). Según el modelo de referencia propuesto por la ONF, la red se divide en tres capas: infraestructura, control y aplicaciones donde cada capa tiene bien especificadas sus funcionalidades, las cuales son descritas con precisión entre otros por (Xia, et al. 2015).

Las SDN se definen como una arquitectura de red dinámica, gestionable, adaptable, de costo eficiente. Lo cual la hace ideal para las altas demandas de ancho de banda y la naturaleza dinámica de las aplicaciones actuales. (de la Torre, Paliza, \& Fleites, 2019). Esta arquitectura desacopla el control de la red y la funcionalidad de reenvío de información permitiendo que el control de la red pueda ser completamente programable logrando que las aplicaciones y servicios de red se abstraigan de la infraestructura de red subyacente (Darabseh, et al. 2015) Esta separación permite que el control de la red pueda ser completamente programable 
logrando que las aplicaciones y servicios de red se abstraigan de la infraestructura de red subyacente (Haleplidis, et al., 2015)

Dado estos antecedentes y debido a que un campus universitario posee características únicas por lo cual tiene diversas necesidades, (Santillán-Lima, Rocha-Jacome, Guerrero-Morejón, Llanga-Vargas, Vásconez-Barrera, Molina-Granja, 2017b) (Santillán, 2013) y basándonos en estas necesidades surge el presente trabajo de investigación propone aplicar SDN en las infraestructuras telecomunicaciones de redes educativas. Y determinar los parámetros para aplicar SDN correctamente en infraestructura de redes educativas

\section{Metodologia.}

Para poder contestar las preguntas de investigación planteadas, se planifica un proceso metodológico dividido en 2 etapas que se detalla a continuación:

Primera etapa: Análisis teórico: en esta fase de la investigación se realizará una recopilación detallada de información, con la finalidad de conceptualizar SDN, los parámetros para aplicar SDN correctamente en infraestructura de redes educativas, mediante el estudio de tesis libros y artículos publicados en varias conferencias y revistas al respecto.

En la tabla 1 se pueden observar con mayor detalle los aspectos considerados para el análisis teórico en la metodología utilizada en la presente investigación.

\section{Tabla 1. Metodología}

\begin{tabular}{|c|c|}
\hline ASPECTO & EXPLICACIÓN \\
\hline $\begin{array}{l}\text { Preguntas a } \\
\text { investigar }\end{array}$ & $\begin{array}{l}\text { Esta investigación busca responder a las siguientes preguntas de } \\
\text { investigación: } \\
\text { ¿Cuáles son los parámetros para aplicar SDN correctamente en } \\
\text { infraestructura de redes educativas? }\end{array}$ \\
\hline $\begin{array}{l}\text { Estrategias de } \\
\text { investigación }\end{array}$ & $\begin{array}{l}\text { Área: Redes de comunicaciones, Seguridad de Redes, } \\
\text { Telecomunicaciones } \\
\text { Propósito de la búsqueda: Determinar los factores que influyen al } \\
\text { aplicar SDN en redes educativas }\end{array}$ \\
\hline $\begin{array}{l}\text { Fuentes de } \\
\text { información }\end{array}$ & Tesis, Artículos científicos, Libros \\
\hline $\begin{array}{l}\text { Motores de } \\
\text { búsqueda }\end{array}$ & Google Scholars, Scielo, Scopus, \\
\hline $\begin{array}{l}\text { Criterios de } \\
\text { búsqueda }\end{array}$ & $\begin{array}{l}\text { "SDN", "Redes educativas y de investigación", "Redes educativas } \\
\text { y de investigación y SND" }\end{array}$ \\
\hline
\end{tabular}


Criterios de Documentos que contienen información sobre "Redes educativas y

Inclusión de investigación", "Redes educativas y de investigación y SND"

Criterios de

Exclusión

Se excluyen los documentos no referentes al ítem anterior

Evaluación del contenido de los criterios

Exactitud, objetividad, cobertura, relevancia de acuerdo con las preguntas de investigación.

Se investiga en la cadena de autores que han realizado contribuciones a los ítems vistos.

Análisis de la Se proporciona una visión general de SDN y se determina los información factores que influyen al aplicar SDN en redes educativas

Segunda etapa: Análisis de los factores que influyen al aplicar SDN en redes educativas Todas estas etapas nos permiten conocer el estado actual de SDN y aportar una referencia a los investigadores.

\section{Resultados.}

En la sociedad actual se prioriza la información como un capital muy importante de las empresas e inclusive de la humanidad, un compendio de criterios sobre el Capital Intelectual nos dice que "está constituido por todos aquellos conocimientos o ideas que poseen los miembros de una empresa y que son puestos en práctica para contribuir a darle ventajas competitivas dentro del mercado en que se desenvuelve". (Edvinsson y Malone, 1998), (Stewart, 1998), (Roos et al, 2001), (Román, 2004). Demostrando de esta manera la importancia no solo de los bienes materiales sino también de los conocimientos e ideas plasmadas en información o datos. Una de las principales fuentes generadoras de conocimiento son las universidades.

Y esta información debe ser transmitida y compartida por lo cual la principal herramienta para realizar esta acción es las telecomunicaciones, dado esto es de vital importancia el conocer el estado actual de las investigaciones y aplicaciones de SDN debido a que es fundamental para los investigadores tener un marco de referencia ya sea para nuevas investigaciones o para tener nuevas aplicaciones de esta tecnología. A la vez comprender el impacto que tiene en nuestra sociedad las redes definidas por software y las redes de comunicaciones.

Al analizar las investigaciones realizadas por Santillán et all. (2017a, 2017b, 2018), encontramos los factores fundamentales que deben ser tomados en cuenta para el diseño de redes de campus universitarios. Así mismo una infraestructura de red educativa posee características únicas (Santillán, 2013, 2017a) por lo cual tiene diversas necesidades no solo de docentes calificados, laboratorios equipados, edificios, personal administrativo, sino 
también es preciso contar con una infraestructura de red (CEAACES, 2014) que pueda ofrecer el acceso a las diversas Tecnologías de la Información y Comunicación. (Santillán Lima, Molina Granja, Vásconez Barrera, Luna Encalada, \& Lozada Yánez, 2018)

Dentro de las necesidades que debe soportar la infraestructura de red de un Campus Universitario podemos encontrar:

- Redes que den acceso a las diversas tecnologías de la información y comunicación.

- Sistema de calificaciones en línea.

- Aulas virtuales.

- Bibliotecas virtuales.

- Aplicaciones propias de cada universidad.

- Internet.

- Telefonía IP.

- Correo institucional.

- Ancho de banda acorde a los servicios y tecnologías que provee el campus de una universidad

- Conectividad (CEAACES, 2014)

- Acceso a estudiantes (CEAACES, 2014)

- Innovación tecnológica (CEAACES, 2014)

Dentro de estas características se destacan las siguientes

Internet: El internet como biblioteca se convierte en una herramienta importante para estudiantes y docentes dando la posibilidad de traer la información necesaria hacia el aula para fortalecer la educación. A su vez el internet al tener gran cantidad de información da a los estudiantes y docentes diferentes perspectivas sobre un tema, "por tanto, la red es un recurso formidable para enriquecer la perspectiva de maestros y alumnos; y el proceso de analizar, valorar, integrar información diversa es la esencia del proceso de construcción de conocimientos" (Adell, 2004)

Espacios virtuales: los espacios virtuales como recurso educativo están penetrando aceleradamente en el campo universitario", podemos inferir y contribuir a ello, constatando en esta investigación que efectivamente existe una penetración y desarrollo continuo de infraestructuras tecnológicas en las universidades, sin embargo, se están descuidando áreas prescindibles como la percepción de utilidad y la facilidad de uso de esas tecnologías, lo que genera no exista una apropiación, originando el desuso de la tecnología que rápidamente se propaga

Las características más relevantes que han puesto en evidencia estos estudios con relación al proceso de aprendizaje en aulas virtuales son: • Una organización menos definida del espacio y el tiempo educativos.

- Un uso más amplio e intensivo de las TIC.

- Planificación y organización del aprendizaje más guiados en sus aspectos globales.

- Unos contenidos de aprendizaje apoyados con mayor base tecnológica. 
- Una forma telemática de llevar a cabo la interacción social.

- Un desarrollo de las actividades de aprendizaje más centrado en el alumnado. (Barberà \& Badia, 2005)

Sistemas académicos: Tradicionalmente el método de evaluación y verificación del aprendizaje y asimilación de conocimientos se lo ha realizado mediante exámenes, evaluaciones, deberes, trabajos, informes y demás; todo esto conduciendo a calificaciones en diferentes escalas que representan el alcanzar o no un conocimiento, estas calificaciones se las llevaban y almacenaban en papeles que se almacenaban en oficinas específicas para ello, ocupando una gran cantidad de espacio y dificultando el acceso a la información. (Santillán et all, 2018)

Santillán analiza desde el punto de vista de los servicios y aplicativos propios de los requerimientos de usuarios en este tipo de redes, pero más allá de dicha visión se deben analizar los requerimientos desde el punto de vista de la red, y más aun de una red implementada en SDN:

- Gestión automatizada de la red: la cual se debe verificar y depurar según sus especificaciones.

- Gestión de actualizaciones de la red: el mantenimiento y las actualizaciones de la red son tareas vitales que requieren cuidado y atención.

- Lenguaje de políticas de red: Se han desarrollado varios lenguajes de políticas de red para redes SDN puras.

- $\quad$ Seguridad: La seguridad es un aspecto muy importante de cualquier red informática. Se han realizado una amplia gama de mecanismos de seguridad.

- Eficiencia energética: La informática y las redes ecológicas se han convertido en un área de muy importante en los últimos años y su aplicación en universidades ayudaría resolver el problema de gasto energético.

- Virtualización de redes: La virtualización ayuda a reducir los costos de la red, al tiempo que mejora el rendimiento y la eficiencia de la red (Afolabi, et all., 2018).

- Controladores SDN distribuidos: Un controlador distribuido debe proporcionar escalabilidad, confiabilidad y simplicidad. El controlador distribuido debería proporcionar una vista coherente de toda la red (Aslan, \& Matrawy, 2016) y una sincronización rápida de los eventos de la red. Direcciones de investigación futuras para controladores SDN distribuidos para las redes SDN híbridas incluyen: ¿Cómo se puede recopilar de manera eficiente la información de topología de los dispositivos heredados, que no están conectados directamente al controlador SDN? ¿Cómo se pueden comunicar los dispositivos heredados con varias instancias de controlador distribuido? ¿Cómo se puede lograr la escalabilidad con controladores distribuidos en redes SDN híbridas? 
- Medidas de red: La medición y la supervisión de la red proporcionan información valiosa para optimizar las operaciones de la red y optimizar el rendimiento de la red (Megyesi, P., Botta, A., Aceto, G., Pescape, A., \& Molnar, S., 2017)

- Calidad de servicio y calidad de la experiencia del usuario QoE. (Abuteir, Fladenmuller y Fourmaux, 2016; Bakhshi y Ghita, 2016; Bozkurt y Benson, 2016) Abordando principalmente los contenidos multimedia y transmisión de video, con el objetivo de optimizar la asignación de ancho de banda para diferentes aplicaciones de red para mejorar la experiencia del usuario.

- Dispositivos IoT, en la universidad inteligente Esta perspectiva por su relevancia está aumentando con el auge del paradigma de IoT. Por lo cual es importante llevar este paradigma a las redes de instituciones universitarias, los dispositivos eventualmente están formando un Internet de las cosas y, en consecuencia, su administración puede considerarse un para la implementación de una red de universidad inteligente

- Seguridad de la red. Se debe proponer un mecanismo de mitigación de ataques de múltiples etapas utilizando SDN.

\section{Conclusiones.}

- Se debe investigar cómo aplicar IoT en las redes Universitarias bajo el paradigma de SDN, lo cual conllevara a tener la universidad automatizada y optimizar los servicios

- El diseño de la infraestructura de comunicaciones de telecomunicaciones no se debe abordar solo desde el punto de vista de los servicios y aplicativos propios de los requerimientos de usuarios en este tipo de redes, sino también se deben analizar los requerimientos desde el punto de vista de la red, y más aun de una red implementada en SDN.

- Los factores que se añaden al diseño de infraestructura de telecomunicaciones al aplicar a una red universitaria son: Gestión automatizada de la red. Gestión de actualizaciones de la red. Lenguaje de políticas de red. Seguridad. Eficiencia energética. Virtualización de redes. Controladores SDN distribuidos. Medidas de red, Calidad de servicio y calidad de la experiencia del usuario QoE. Dispositivos IoT, en la universidad inteligente, Seguridad de la red.

\section{Referencias bibliográficas.}

Abuteir, R. M. , Fladenmuller, A. , \& Fourmaux, O. (2016). An SDN approach to adaptive video streaming in wireless home networks. In 2016 International wireless communications and mobile computing conference (IWCMC) (pp. 321-326)

Alshnta, A. M., Abdollah, M. F., \& Al-Haiqi, A. (2018). SDN in the home: A survey of home network solutions using software defined networking. Cogent Engineering, 5(1), 1-40. doi:10.1080/23311916.2018.1469949 
Aslan, M., \& Matrawy, A., (2016) "Adaptive consistency for distributed SDN controllers," in Proc. IEEE Int. Telecommun. Network Strategy and Planning Symp. (Networks), pp. 150-157.

AutoBAHN, (2020),

recuperado

de: http://geant3.archive.geant.net/service/autobahn/pages/home.aspx.

Bakhshi, T. , \& Ghita, B. (2016). User-centric traffic optimization in residential software defined networks. In 2016 23rd International conference on telecommunications (ICT) (pp. 1-6)

Bozkurt, I. N. , \& Benson, T. (2016). Contextual router: Advancing experience-oriented networking to the home. In Proceedings of the symposium on SDN research (p. 15: 115:7).

CEAACES. (2014). Modelo institucional de pregrado posgrado. Quito.

Charbonneau, N., \& Vokkarane, V. M., (2012) "A survey of advance reservation routing and wavelength assignment in wavelength-routed WDM networks", IEEE Commun. Surveys Tuts., vol. 14, no. 4, pp. 1037-1064, 4th Quart.

Chergarova, V. (2020). Factors Affecting Software Defined Networking Adoption by Research and Educational Networks.

Chica, J. C. C., Imbachi, J. C., \& Botero, J. F. (2020). Security in SDN: A comprehensive survey. Journal of Network and Computer Applications, 102595.

De la Torre, D. I., Paliza, F. Á., \& Fleites, A. R. (2019). Combinación de mecanismos MPLS en una arquitectura SDN. Telemática, 18(1), 1-10.

Degermark, M., Köhler, T., Pink, S., \& Schelen, O., (1995) "Advance reservations for predictive service" in Network and Operating Systems Support for Digital Audio and Video, Berlin, Germany: Springer, pp. 1-15.

Dyer, J. (2009). The Case for National Research and Education Networks (NRENs). TERENA Networking Conference (TNC)

Edvinsson, L. y Malone, M. (1998). “El capital intelectual”. Bogotá: Norma.

ESnet (2017), recuperado de: http://www.es.net/.

ESnet's OSCARS with FloodLight, (2020) recuperado de: https://github.com/hsr/oscars-gui.

Géant, (2020) recuperado de: https://www.geant.org/.

Haleplidis, E., Pentikousis, K., Denazis, S., Salim, J. H., Meyer, D., \& Koufopavlou O. (2015) "Software-defined networking (SDN): Layers and architecture terminology," 2070-1721. 
Hamdan, M., Hassan, E., Abdelaziz, A., Elhigazi, A., Mohammed, B., Khan, S., ... \& Marsono, M. N. (2020). A comprehensive survey of load balancing techniques in software-defined network. Journal of Network and Computer Applications, 102856.

Latif, Z., Sharif, K., Li, F., Karim, M. M., Biswas, S., \& Wang, Y. (2020). A comprehensive survey of interface protocols for software defined networks. Journal of Network and Computer Applications, 156, 102563.

Li, W., Meng, W., \& Kwok, L. F. (2016). A survey on OpenFlow-based Software Defined Networks: Security challenges and countermeasures. Journal of Network and Computer Applications, 68, 126-139.

McKeown, N., Anderson, T., Balakrishnan, H., Parulkar, G., Peterson, L., Rexford, J., ... \& Turner, J. (2008). OpenFlow: enabling innovation in campus networks. ACM SIGCOMM Computer Communication Review, 38(2), 69-74.

Megyesi, P., Botta, A., Aceto, G., Pescape, A., \& Molnar, S. (2017) “Challenges and solution for measuring available bandwidth in software defined networks," Computer Communications, vol. 99, no. Supplement C, pp. 48-61.

Nayyer, A., Sharma, A. K., \& Awasthi, L. K. (2019). Issues in software-defined networking. In Proceedings of 2nd International Conference on Communication, Computing and Networking (pp. 989-997). Springer, Singapore.

Román, N. (2004). “Capital intelectual: generador de éxito en las empresas”. Visión Gerencial, (2), 67-79.

Roos, J., Roos, G., Dragonetti, N. y Edvinsson, L. (2001). “Capital Intelectual”. Paidós. Buenos Aires

Santillán Lima, J. C., Llanga Vargas, A., \& Chafla, G. (2017a). Metodología para diseño de infraestructura de telecomunicaciones para campus universitarios medianos, caso La Dolorosa-UNACH. Revista Ciencia UNEMI, 10.

Santillán-Lima, J. C. (2013). Diseño de una infraestructura de telecomunicaciones que optimice el acceso a los servicios para el creciente tráfico de datos del Campus La Dolorosa de la UNACH (Tesis de maestría). Pontificia Universidad Católica del Ecuador, Quito.

Santillán-Lima, J. C., Rocha-Jacome, C., Guerrero-Morejón, K., Llanga-Vargas, A., Vásconez-Barrera, F., Molina-Granja, F., (2017b). "EL IMPACTO DE LOS SERVICIOS DE TELECOMUNICACIONES Y LAS TICS EN LAS NECESIDADES DE LA EDUCACIÓN SUPERIOR”. IV Congreso Internacional de Ciencia Tecnología Innovación y Emprendimiento CITE 2017. Universidad Estatal de Bolívar, Guaranda.

Santillán Lima, J. C., Molina Granja, F. T., Vásconez Barrera, M. F., Luna Encalada, W. G., \& Lozada Yánez, R. M. (2018). Requerimientos y diseño de infraestructura de redes para campus universitarios. 
Stewart, T. (1998). "La nueva riqueza de las organizaciones: el capital intelectual”. Granica, Buenos Aires.

Thimmaraju, K., Shastry, B., Fiebig, T., Hetzelt, F., Seifert, J. P., Feldmann, A., \& Schmid, S. (2018). Taking control of sdn-based cloud systems via the data plane. In Proceedings of the Symposium on SDN Research (pp. 1-15).

Xia, W., Wen, Y., Foh, C. H., Niyato, D., \& Xie, H. (2015). A survey on SoftwareDefined Networking. IEEE Communication Survey \& Tutorial, 17(1), 27-51. 
PARA CITAR EL ARTÍCULO INDEXADO.

Bone Andrade, M. F., Rodríguez Vizuete, J. D., Sosa Calero, S. M., \& Núñez Freire, L. A. (2021). Aplicaciones de SDN en infraestructura de redes educativas . Ciencia Digital, 5(1), 219-231. https://doi.org/10.33262/cienciadigital.v5i1.1539

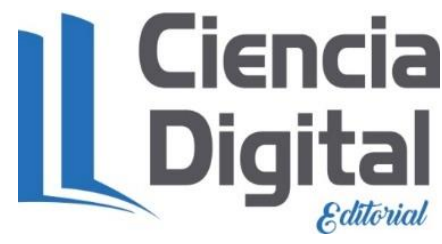

El artículo que se publica es de exclusiva responsabilidad de los autores y no necesariamente reflejan el pensamiento de la Revista Conciencia Digital.

El artículo queda en propiedad de la revista y, por tanto, su publicación parcial y/o total en otro medio tiene que ser autorizado por el director de la Revista Conciencia Digital.
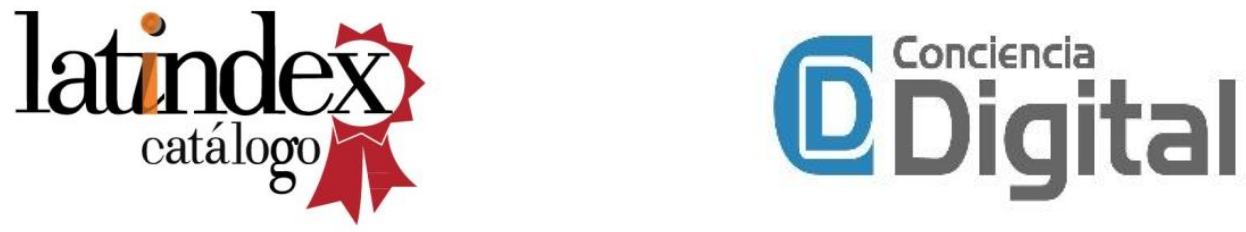